\title{
La organización flexible y su influencia en la implantación de prácticas de alto rendimiento: un estudio empírico
}

\author{
Santiago GUTIÉRREZ BRONCANO \\ Profesor Universidad de Castilla La Mancha (UCLM) \\ Departamento de Administración de Empresas \\ santiago.gutierrez@uclm.es \\ Mercedes RUBIO ANDRÉS \\ Profesora Universidad CEU San Pablo \\ Departamento de Empresa \\ mrubio@ceu.es \\ Juan Nicolás MonTOYA MONSALVE \\ Profesor Universidad Nacional de Colombia (Sede Manizales) \\ nmontoyamo@unal.edu.co
}

Recibido: 05-02-2011

Aceptado: 03-07-2011

\section{RESUMEN}

Las empresas necesitan ser flexibles para adaptarse al entorno y poder competir. La flexibilidad del comportamiento del empleado representa un indicador de la flexibilidad de la empresa. Cuanto más flexibles sean las prácticas de gestión de recursos humanos, la flexibilidad global de la empresa se incrementará. Por ello, en el presente estudio se trata de determinar si las prácticas de alto rendimiento apoyadas en variables organizativas que mejoren la flexibilidad de la empresa, influyen de manera 
positiva en el compromiso de los empleados. Esta relación se analiza en empresas que se encuentran bien valoradas por el Great Place to Work debido a las prácticas de recursos humanos que utilizan y donde se observa, mediante el análisis cluster realizado, la existencia de dos grandes grupos de empresas: uno en el que las empresas utilizan prácticas de alto rendimiento pero se encuentran en una fase inicial en el logro de un alto compromiso de sus empleados y donde su organización no es demasiado flexible; y un segundo grupo que llevando a cabo prácticas de alto rendimiento consigue mejores resultados en cuanto a compromiso y que presenta una estructura organizativa muy flexible.

Palabras clave: Flexibilidad organizativa; compromiso empleados; prácticas alto rendimiento.

\title{
Organizational flexibility impact on high performance human resources practices: empirical study
}

\begin{abstract}
Companies need to be flexible to adapt to the competitive environment. The flexibility of the employee's behaviour is an indicator of the flexibility of the company. The more flexible the management practices of human resources, the overall flexibility of the company will increase. Therefore, this paper is to determine whether high-performance practices supported by organizational variables that improve the flexibility of the company, have a positive impact on employee commitment. We analise this relationship in companies that are highly valued by the Great Place to Work in terms of human resource practices. Through cluster analysis, we conclude the existence of two groups of companies: one in which companies use high-performance practices but are at an initial stage achieving a high employee commitment although the organization is not too flexible, and a second group that conducted high-performance practices get better results in terms of commitment having a very flexible organizational structure.
\end{abstract}

Keywords: Organizational flexibility; employee commitment; high performance practices.

Sumario: 1. Introducción. 2. Prácticas de Alto Rendimiento. 3. Diseño organizativo flexible. 4. Compromiso con los empleados 5. Hipótesis 6. Metodología 7. Conclusiones. Bibliografía.

\section{INTRODUCCIÓN}

El concepto de flexibilidad organizativa, permite a las empresas que puedan adaptarse rápidamente y llevar a cabo grandes cambios en sus organizaciones (Bahrami, 1992, Evans, 2001). La flexibilidad supone para la empresa poseer una habilidad para reaccionar ante las incertidumbres del entono, a través de un conjunto de recursos y capacidades que le permitan afrontar la situación de manera adecuada (Volberda, 1996)

La dificultad principal se encuentra en determinar cuáles son los factores que permiten afirmar que una empresa es flexible. Las principales investigaciones determinan que dichos factores han de venir definidos primero por el diseño estructu- 
ral (Englehardt y Simmons, 2002), y segundo, por una capacidad de gestión enfocada hacia la flexibilidad de la empresa (Volberda, 1997).

Al igual que es necesario referirnos a la flexibilidad empresarial (MacDuffie, 1995; William et al, 1991; Snow y Snell 1993), lo es cada vez más cuando nos referimos a la gestión estratégica de los recursos humanos. Los investigadores han abogado por la flexibilidad en los recursos humanos para ayudar a la organización a adaptarse a un entorno complejo y dinámico (Lengnick-Hall y Lengnick-Hall, 1988; Kerr y Jackofsky 1989; Milliman et. al. 1991; Snow y Snell, 1993).

La flexibilidad de los recursos humanos es la base para el desarrollo del resto de dimensiones de flexibilidad organizativa (Karuppan, 2004; Upton, 1995; Wright y Snell, 1998) y puede constituirse en una capacidad organizativa que suponga una ventaja competitiva sostenible a partir de la mejora de la eficacia organizativa (Bhattacharya et al., 2005; Wright y Snell, 1998). Desde esta perspectiva, la flexibilidad de recursos humanos ha sido percibida como una capacidad que ayuda a la organización a interaccionar con el exterior (Snell, Youndt y Wright, 1996 y Milliman, et. al 1999). Las prácticas de recursos humanos pueden incorporar criterios de flexibilidad tanto en la coordinación como en las habilidades y comportamiento entre los empleados (Wright y Snell, 1998).

Existen varias clasificaciones de la flexibilidad de los recursos humanos, confrontándose unas con otras: flexibilidad funcional y flexibilidad numérica (Atkinson 1984; Smith 1997; Hunter et al. 1993), flexibilidad interna y externa (Cappelli y Neumark 2001), cultura del clan y cultura hacia el mercado (Ouchi, 1980), flexibilidad dinámica y flexibilidad estática (Colclough y Tolbert 1992; Deyo 1997), y la organización centrada en el trabajo frente a la centrada las relaciones laborales (Tsui et al. 1995).

La flexibilidad funcional hace referencia a la capacidad de los trabajadores para asumir distintas actividades. La flexibilidad numérica es la capacidad de la empresa para ajustar el volumen y la carga de trabajo en función de la demanda (Martínez et. al. 2011). Por ello, en este trabajo, nos centramos en los dos primeros tipos de flexibilidad, porque son los que están más directamente relacionados con el diseño organizativo y tienen un efecto en el compromiso de los empleados

La flexibilidad empresarial y de recursos humanos están relacionadas. Cuanto más flexibles sean las prácticas de gestión de recursos humanos, la flexibilidad global de la empresa se incrementará. De hecho, la flexibilidad del comportamiento del empleado representa un indicador de la flexibilidad de la empresa. La empresa, puede lograr flexibilidad mediante la coordinación de comportamientos entre individuos y grupos (Ordóñez 2005).

Investigaciones recientes sobre la flexibilidad de organización han llevado a la conclusión de que existe una correlación entre las prácticas de recursos humanos de alto rendimiento y la flexibilidad funcional. En los últimos años se ha prestado atención a las prácticas que se han diseñado para proporcionar a los empleados las habilidades, los incentivos, y la capacidad de tomar decisiones con mayor nivel de 
responsabilidad, a través de una mayor flexibilidad con el objetivo de mejorar el rendimiento empresarial. Estas nuevas prácticas han sido llamadas, según el caso, organizaciones de alto rendimiento en el trabajo (Appelbaum et al 2000;. Osterman 2000), prácticas de recursos humanos (Gittleman et al. 1998), los sistemas de participación de los trabajadores (Cotton 1993), los sistemas de producción flexible (MacDuffie 1995), prácticas de gestión de recursos humanos (Delaney y Huselid 1996), de alto de compromiso (Walton, 1985) y la gestión de alta participación (Lawler, 1988; Madera 1999).

Estos sistemas de incrementar la flexibilidad funcional de una organización, permite a los trabajadores a participar en la toma de decisiones, trabajar en equipo y mejorar su compromiso con la organización, entre otras cosas, además de vincular sus compensación con el desempeño de la organización, proporcionando a los empleados habilidades múltiples, de modo que puedan ser redistribuidos con relativa rapidez de un tarea a otra (véanse las revisiones de Appelbaum y Batt, 1994; OCDE, 1999; Wood 1999), lo que genera sin duda una mejora de la flexibilidad de la organización.

Derivado de todo lo anterior, nos planteamos las siguientes preguntas de investigación: por un lado, sabiendo que las prácticas de alto rendimiento otorgan flexibilidad, ¿se podrá determinar la relación entre prácticas de alto rendimiento y compromiso con la organización?; y por otro, debido a que la conducta del empleado también se ve incentivada por los principios y relaciones organizativas, ¿serán los modelos organizativos flexibles, aquellos que favorezcan el incremento del compromiso por parte de los empleados?.

Por tanto, el objetivo que nos planteamos en nuestra investigación es examinar cómo la organización flexible interviene de una manera determinante en la relación entre las prácticas de alto rendimiento y el compromiso de los empleados. De esta manera, si pretendemos mejorar el compromiso de los empleados utilizando prácticas de alto rendimiento, cuándo nos apoyemos en variables organizativas flexibles se logrará un mayor grado de consecución.

Para conseguirlo, en primer lugar se han establecido las prácticas de alto rendimiento que la literatura plantea y su repercusión en el compromiso de los empleados en segundo lugar se han establecido las principales variables organizativas que permiten mayor flexibilidad al diseño organizativo; y por último se ha analizado la relación existente entre el diseño organizativo flexible junto con las prácticas de alto rendimiento, y su impacto en el compromiso de los empleados.

\section{PRÁCTICAS DE ALTO RENDIMIENTO}

En la literatura encontramos diferentes trabajos que establecen como superiores, en cuanto a su efectividad, un conjunto de prácticas de recursos humanos, que son denominadas como prácticas de alto rendimiento (Arthur, 1994; Huselid, 1995; 
Delaney y Huselid, 1996; Ichniowski, Shaw y Prennushi, 1999). El sistema de gestión de recursos humanos a través de estas prácticas de alto rendimiento, ha sido difundido y puesto en práctica en el mundo empresarial por un alto porcentaje de empresas (Wood y Albanese, 1995; Osterman, 2000). Huselid (1995: 635), define estas prácticas como aquellas acciones de recursos humanos que "pueden reforzar los conocimientos, habilidades y destrezas de los empleados actuales y potenciales, aumentar su motivación (...) y mejorar la retención de los empleados cualificados". $\mathrm{Y}$ aunque existen investigaciones que evidencian el poco acuerdo que existe sobre cuáles son estas prácticas (Ferris et al, 1999; Roche, 1999), en general se considera que las empresas que desarrollan en mayor medida estas prácticas son las que presentan una estrategia de gestión de recursos humanos más avanzada o proactiva (Arthur, 1994; Batt, 2000; Ordiz, 2002).

Al contrario que otras prácticas de recursos humanos más tradicionales, las prácticas de alto rendimiento fomentan el compromiso de los trabajadores con la organización y con sus objetivos (Arthur, 1994; Kofman y Senge, 1993), así como la mejora de las capacidades de los trabajadores (Bayo y Merino, 2002) y consiguiendo una mayor implicación del trabajador en la empresa (Sanz y Sabater, 2002).

El principal problema que se plantea, respecto a la utilización de éstas prácticas, es que las investigaciones que han analizado la relación entre las prácticas de alto rendimiento y el rendimiento empresarial, no han obtenido resultados concluyentes (ej. Roca, Escrig y Bou, 2002; Sanz y Sabater, 2002). Además, una empresa no obtiene una mayor rentabilidad de las prácticas de gestión de recursos humanos, per sé, sino que esto depende de la articulación de las mismas (Huselid, 1995), de los principios y relaciones organizativas que sustentan, desarrollan e incentivan a los trabajadores a poner realmente en práctica sus conocimientos. Es en esta línea, donde el grado de compromiso (actitud) que la dirección muestra hacia el empleado se pondrá de manifiesto en una determinada estructura organizativa y social adecuada para que los empleados desarrollen y pongan en práctica al máximo sus conocimientos, habilidades y destrezas y podamos considerarlo como un recurso valioso, escaso y no imitable (Wright et al, 1994; Wright et al, 1995; Becker y Gerhart, 1996; Kamoche, 1996; Delery, 1998). Esta sería la forma en la que las prácticas de recursos humanos estarían integradas en la visión estratégica de la organización proporcionando una visión global o de sistema (Roche, 1995; Roca, Escrig y Bou, 2002), enfatizando la autonomía de los trabajadores sobre su trabajo (Arthur, 1994), favoreciendo de esta manera la experimentación y promoviendo la comunicación interna y la transferencia de conocimiento (Truss et al, 1997).

Si nos centramos en la gestión de recursos humanos y la consideramos como un factor que dispone de gran potencial para convertirse en un elemento innovador y por lo tanto en generador de ventaja competitiva sostenible para la empresa, encontramos principalmente dos modelos contrapuestos. El primero de ellos lo podemos considerar como el más tradicional, basado principalmente en el control estricto y la subordinación de los empleados (Sashkin, 1984). Y el segundo, bastante más 
innovador, es el que se ha denominado como gestión de alto rendimiento (Huselid, 1995; Céspedes et al., 2005; Beltrán et al, 2008; Taylor et al., 2008), de alto compromiso (Lawler, 1992; Bayo y Merino, 2002, González y Nieto, 2007; Martí, 2008) o incluso de alta implicación (Arthur, 1994; Guthrie, 2001; Guthrie et al., 2002, Ordiz y Fernández, 2003; Zatzick e Iverson, 2006; Mohr y Zoghi, 2008; Word y Menezes, 2008). Truss et al. (1997) mantienen que ambos modelos están basados en los planteamientos extremos sobre comportamiento humano propuestos por McGregor (1960). Por lo tanto, el modelo de gestión de alto rendimiento estaría basado en la teoría Y, por ello enfatiza la autonomía y el autocontrol de los empleados sobre su trabajo (Guest, 1987) y se centra en el reconocimiento de las necesidades de los individuos (Kofman y Senge, 1993; Truss et al., 1997), la comunicación continua (Truss et al, 1997; Walton, 1985) y la importancia de integrar los recursos humanos dentro de la estrategia (Roche, 1999; Wood y Albanese, 1995).

Aunque los diferentes autores y trabajos que han analizado estas prácticas de alto compromiso no consiguen ponerse de acuerdo en determinar cuáles son, sí que podemos afirmar que todas ellas tienen una orientación común, que según Guthrie (2001: 181) consiste en "enfatizar el uso de un sistema de prácticas directivas que dota a los empleados de destrezas, información, motivación y discrecionalidad, resultando una fuerza de trabajo que es fuente de ventaja competitiva".

La mayoría de los trabajos en este campo de estudio, han analizado la relación entre el sistema de gestión de recursos humanos, los resultados y la estrategia competitiva (Delery y Doty, 1996, Lee y Miller, 1999; Lengnick-Hall y Lengnick-Hall, 1988, Schuler y Jackson, 1987, Valle et al., 2000; Danvila y Sastre, 2007) y todos ellos han defendido la necesidad de un ajuste entre el sistema de gestión y la estrategia para la consecución de resultados. El problema que se plantea es que existe poca evidencia empírica que sugiera que este ajuste sea necesario o beneficioso para la organización (Delery, 1998; Roche, 1999) y que la mera implementación de determinadas prácticas de recursos humanos, por muy innovadoras que éstas sean, no garantiza ni siquiera la mejora en los resultados empresariales. Las hipótesis sometidas a contraste empírico, han establecido asociaciones de diverso signo, sin que existan resultados concluyentes al respecto (Huselid, 1995; Delery y Doty, 1996). Además, encontramos que algunos autores cuestionan la metodología utilizada para determinar la gestión centrada en el compromiso (Delery, 1998; Roca et al., 2002).

Las prácticas de recursos humanos identificadas y vinculadas al alto rendimiento son de una gran variedad (Becker y Gerhart, 1996), existiendo diversos enfoques sobre aquellas prácticas que deberían incluirse o no como de alto rendimiento (Word, 1999).

Ichniowski et al., (1996) establecen la flexibilidad laboral, el trabajo en equipo, la remuneración contingente, el empowerment, la seguridad en el empleo, etc., como las prácticas que generan mayor compromiso entre los empleados. Este enfoque parte de la base de que el efecto que se produce entre las capacidades y la mo- 
tivación tiene un carácter multiplicador en el valor creado en la organización (Ulrich, 1998). Marchington y Grugulis (2000) plantearon un modelo que toma como punto de referencia las siete prácticas identificadas por Pfeffer $(1994,1998)$ como generadoras de éxito: seguridad en el empleo, contratación selectiva de personal, trabajo en equipo, compensación contingente, formación extensiva, reducción de diferencias de estatus y transferencia de información. No hay que olvidar que básicamente, se trata de un planteamiento de recursos humanos orientado el empleado y que implica la participación activa de éstos en la toma de decisiones, por lo que ha de proporcionarles los recursos y el apoyo organizativo necesario para ello (Roca et al., 2002).

Aunque las investigaciones recientes evidencian el poco acuerdo sobre qué prácticas componen un sistema coherente de gestión de recursos humanos orientado hacia el rendimiento, compromiso e implicación del trabajador (Ferris et al, 1999; Roche, 1999), podemos observar cómo existe una alta coincidencia entre distintos autores en determinadas prácticas de alto rendimiento que determinan y que se describen en la Tabla 1.

La puesta en práctica de todas estas acciones de recursos humanos tiene un impacto en el empleado ya que aumenta su capacidad, su motivación y el compromiso con la organización. (Pfeffer, 1998; Sánchez, 2004; Lings, 2004; Barroso et al., 2005; Tsai y Wu, 2006).

Aún así, si seguimos el enfoque contingencial, para que las políticas de recursos humanos sean efectivas deben ser consistentes con otros aspectos organizativos (Delery y Doty, 1996). Biard y Meshoulam (1988) ya mencionaban tanto el ajuste interno, prácticas de recursos humanos relacionadas entre ellas; como el ajuste externo, identificado fundamentalmente en relación con la estrategia implantada por la empresa.

Para el presente estudio, el ajuste de las prácticas de alto rendimiento busca no sólo un modelo integrado de prácticas de recursos humanos coherentes entre sí, sino además, congruentes con la existencia de un diseño organizativo flexible que favorezca la consecución de un mayor grado de compromiso por parte del trabajador con la organización. 
Tabla 1: Prácticas de alto rendimiento

\section{PRÁCTICAS DE}

ALTO RENDIMIENTO

\section{Diseño de puestos amplios}

\section{Reclutamiento selectivo}

acordes con los requisitos
del puesto. Se analizan
conductas pasadas, pre-
sentes y futuras

Contrataciones fijas Estabilidad en el trabajo

\section{Formación extensi- va \\ Valoración del desempeño} evitando la temporalidad en el trabajo

\section{Puestos de trabajo am-} plios, especialización vertical y horizontal baja Criterios de reclutamiento y selección definidos y acordes con los requisitos conductas pasadas, preen futuras

Gran importancia e inversión en formación y desarrollo del empleado. Formación a todos los empleados continua y evaluada. Incentivos para las nuevas habilidades Sistemas de evaluación basados en los resultados

\section{Compensación contingente}

Políticas salariales centradas en los resultados individuales y a los del grupo. Retribución por encima de la media del mercado. Incentivos tangibles e intangibles, flexibles, participativos y públicos.

\section{PRINCIPALES AUTORES E INVES- TIGACIONES}

Schuler y Jackson (1987); Blacburn y Rosen (1993); O'Dell (1996); Capelli y Crocker-Hefter (1996)

Guest (1987), Arthur (1994), Pfeffer (1994), Huselid (1995); MacDuffie (1995); Youndt et al., (1996); Kim (1998); Wood y Menezes (1998); Roche (1999); Bae y Lawler (2000); Bayo y Merino (2001); Williams (2001); Ordiz y Fenández (2003); Céspedes et al., (2005); Beltrán et al., (2008)

Pfeffer (1994); Nonaka y Takeuchi (1995); Argyris y Schön (1996); Delery y Doty (1996); Bae y Lawler (2000); Bayo y Merino (2001); Ordiz y Fernandez (2003); Wood y Menezes (2008)

Leonard-Barton (1992); Schuler y Jackson (1987a), Blacburn y Rosen (1993); Oackland (2004); Danvila y Sastre (2009); Danvila et al. (2009)

Guest (1987); Schneider y Bowen (1993); Osterman (1994); Pfeffer (1994); Huselid (1995); Huselid y Becker (1996); Delery y Doty (1996); Huselid et al., (1997); Wood y Menezes (1998); Capelli y Neumark (2001); Bayo y Merino (2001); Richard y Jonson (2001); Gutrie et al., (2002); Roca et al., (2005); Mohr y Zoghi (2008); Beltrán et al., (2008)

Guest (1987); Blackburn y Rosen (1993); Arthur (1994); Pfeffer (1994); Powell et al., (1994); Huselid (1995); Delaney y Huselid (1996); Delery y Doty (1996); Wood y Menezes (1998); Boxall y Steeneveld (1999); Lee et al., (1999); Roche (1999); Bae y Lawler (2000); Capelli y Neumark (2001); Bayo y Merino (2001); Gutrie et al., (2002); Ordiz y Fernandez (2003); Céspedes et al., (2005) Zatzick e Iverson (2006); Wood y Menezes (2008); Beltrán et al., (2008) 
Tabla 1: Prácticas de alto rendimiento (continuación).

$\begin{array}{ll}\text { Discrecionalidad } & \text { Otorgar al puesto de traba- } \\ \text { Elevada } & \text { jo y al propio empleado de } \\ & \text { gran autonomía para } \\ & \text { determinar su propia } \\ & \text { conducta y normas en el } \\ & \text { trabajo. Descentralización } \\ & \text { y menor especialización } \\ & \text { vertical. }\end{array}$

\begin{tabular}{|c|c|}
\hline Promoción Interna & $\begin{array}{l}\text { Grandes oportunidades de } \\
\text { desarrollos profesionales } \\
\text { en la empresa a través de } \\
\text { un sistema formal de } \\
\text { carreras profesionales }\end{array}$ \\
\hline
\end{tabular}

Trabajo en equipo Incentivo y fomento del trabajo en equipo, alto compromiso entre los miembros y baja rotación.
Schuler y Jackson (1987a y b); Blackburn y Rosen (1993); Schneider y Bowen (1993); Arthur (1994); Pfeffer (1994); Huselid (1995); Cardy y Robbins (1996); Delery y Doty (1996); O,Dell (1996); Pfeffer (1999); Roche (1999); Bayo y Merino (2001); Richard y Johnson (2001); Gutrie et al., (2002); Ordiz y Fernández (2003); Roca et al., (2005); Mohr y Zoghi (2008).

Schneider y Bowen (1993); Arthur (1994); Pfeffer, (1994); Huselid (1995); Cardy y Robbins (1996); Delery y Doty (1996); Roche (1999); Bayo y Merino (2001); Richard y Jonson (2001); Guthrie et al., (2002); Ordiz y Fernández (2003); Roca et al., (2005); Mohr y Yoghi (2008)

Arthur (1994); Osterman (1994); Pfeffer (1994); Huselid y Becker (1996); O,Dell (1996); Huselid et al., (1997); Wood y Menezes (1998); Bae y Lawler (2000); Capelli y Neumark (2001); Bayo y Merino (2001); Richard y Johnson (2001); Guthrie et al., (2002); Zatzick e Iverson (2006); Mohr y Zoghi (2008)

Guest (1987); Schneider y Bowen (1993); Arthur (1994); Pfeffer (1994); Huselid y Becker (1996); Huselid et al., (1997); Wood y Menezes (1998); Roche (1999); Capelli y Neumark (2001); Bayo y Merino (2001) Richard y Johnson (2001); Guthrie et al., (2002); Ordiz y Fernández (2003); Roca et al., (2005); Zatzick e Iverson (2006); Mohr y Zoghi (2008)

\section{Seguridad en el empleo}

Elevada seguridad en los puestos de trabajo. Personal especializado para la mejora en la seguridad laboral

Fuente: Adaptado de Martí (2008)

\section{Schuler y Jackson (1987 a y b); Blackburn y} Rosen (1993) 


\section{DISEÑO ORGANIZATIVO FLEXIBLE}

Hoy en día, el enfoque clásico del diseño organizativo ha ido dando paso a una organización de este tipo, convirtiéndose en algo esencial para el éxito y comenzando a ser la forma más predominante de organización (Evans, 1993; Hastings, 1993; Jarillo, 1993; Castells, 1996; Nohria y Ghoshal, 1997; Schoemaker, 1998).

Volberda (1997) define la forma organizativa flexible como aquélla que posee una alta flexibilidad, tanto organizativa como productiva, que le permite ejercer cierto grado de control en entornos cambiantes.

Si partimos de la revisión de la literatura existente sobre diseño organizativo flexible (Volberda, 1999; Englehardt y Simmnos, 2002, Hage y Dewar, 1993; Nicholson et. al.,), es de suponer que cuanto mayor es el nivel de decisión formal y las decisiones se toman de manera más centralizada, menos flexible será la organización. Por tanto, un menor grado de centralización y formalización de la toma de decisiones, a través por ejemplo de una mayor autonomía y una estructura más plana, son necesarios para adaptarse rápidamente a los cambios del entorno.

La medición de la flexibilidad organizativa se centra por tanto, previamente en el estudio de sus principales variables organizativas. Y siguiendo a Walton (1981), Pennings (1973), Sathe (1978) y Ford (1979), encontramos diferentes dimensiones y medidas en tres ámbitos de la estructura organizativa: centralización, formalización, y especialización (Sánchez, 2002).

En el primer ámbito, la centralización analiza cómo se toman las decisiones que afectan a la organización (Pugh et.al. 1968), y cómo el poder se distribuye entre los diversos puestos de trabajo (Hage y Aiken, 1967). Supone el estudio de variables como las propuestas por Pugh, et. al (1968): Autonomía, Ámbito de control del director, Número de supervisores directos y ratio de supervisión/trabajador; o por Hage y Aiken (1967) y Perrow (1970): Participación personal en la toma de decisiones, Jerarquía de autoridad, jerarquía de control y participación departamental en la toma de decisiones.

La formalización es definida como la medida en que las reglas, procedimientos, instrucciones y comunicaciones son establecidas (Pugh, et. al. 1968), recoge la estructuración de actividades (formalización), la codificación del trabajo (Hage y Aiken, 1967), el grado de control en el cumplimiento de las reglas y procedimientos (Perrow, 1970) y nivel de comunicación escrita (Hage y Aiken, 1967).

La especialización es entendida como la medida en que las funciones están divididas según las áreas funcionales (Sathe, 1978), lo que supone el estudio de variables como la diversidad de funciones, su número, el número de categorías laborales y la división del trabajo.

Agrupando por tanto estas variables podemos establecer las características necesarias para considerar una organización flexible, tal y como se muestra en la Tabla 2. 
Tabla 2: Variables de diseño organizativo

\begin{tabular}{|l|c|}
\hline Centralización & $\begin{array}{l}\text { Nivel a alcanzar en la organiza- } \\
\text { ción flexible }\end{array}$ \\
\hline Autonomía del empleado & Alto \\
\hline Ámbito de control del director & Bajo \\
\hline Número de supervisores directos & Bajo \\
\hline Ratio de supervisión/trabajador & Alto \\
\hline Participación del personal en la toma de decisiones & Alto \\
\hline Jerarquía de autoridad & Baja \\
\hline Jerarquía de control & Baja \\
\hline Participación departamental en la toma de decisiones & Alta \\
\hline Formalización & Baja \\
\hline Formalización (estructuración de actividades) & Bajo \\
\hline Nivel de comunicación escrita & Bajo \\
\hline Número de Reglas y Procedimientos & \\
\hline Especialización & Alto \\
\hline Diversidad de funciones y número & Bajo \\
\hline Número de categorías laborales & Bajo \\
\hline División del trabajo & \\
\hline
\end{tabular}

Fuente: Elaboración propia.

Estos modelos denominados como "organización flexible" permiten a sus individuos y a los equipos gestionar su propio trabajo y talento como una práctica habitual, lo que supone aumentar el potencial de innovación y creatividad dentro de la organización (Schoemaker, 2003).

En definitiva, estructuras descentralizadas que permiten tomar decisiones a todos los niveles y que se dirigen a una forma más flexible de organización ( $\mathrm{McNabb}$ y Whitfield, 1998).

\section{COMPROMISO DE LOS EMPLEADOS}

En el momento actual, que está presente un entorno competitivo, en el que prima la flexibilidad, la efectividad de la gestión de los recursos humanos se evaluará en función del compromiso de los empleados. Este compromiso aparece en la literatura de manera frecuente, como un resultado específico de cualquier proceso de gestión de recursos humanos y como criterio clave para juzgar dicha gestión (Iles, Foster y Tinline, 1996). Para analizar el compromiso organizativo con los empleados, es preciso identificar las dimensiones que lo determinan: compromiso afectivo, continuo, normativo (Meyer y Allen, 1997), o psicológico, de intercambio y de atribución (Bayona, Goñi y Madorrán, 1999). De cada dimensión, se desprenden una serie de elementos, como la identificación con los objetivos y valores de la organización o el deseo de contribuir para que la empresa alcance sus metas y objetivos. 
El compromiso se refiere al grado en el que el empleado se identifica con una determinada organización y sobre todo con sus objetivos, de su deseo de permanecer en la compañía (Blau y Boal, 1987) y de su participación activa en ella (Davis y Newstron, 2000). Por lo que para su medición, algunos autores aconsejan observar el compromiso organizativo como una actitud de los directivos y proponen un análisis en profundidad de los principios que rigen la verdadera política (Delery, 1998 y Roca et al., 2002) utilizando escalas derivadas de la psicología industrial.

Para ello, analizan el compromiso organizacional como un proceso de intercambio social entre la dirección y los trabajadores (Eisenberger et al., 1986). Según este autor, el intercambio se basa en la norma de la reciprocidad (Gouldner, 1960), cuando el empleado perciba que la empresa tiene en consideración sus necesidades, este es proclive a trabajar con mayor dedicación (Bishop et al., 2000; Eisenberger et al., 1986, Wayne et al., 1997).

Este compromiso que se genera en los empleados hace que se orienten hacia un comportamiento positivo que contribuya de una forma más efectiva a la consecución de los objetivos organizativos y por ende se mejoran los resultados empresariales (Ordiz y Fernández, 2003; Ahmed, Rafiq y Saad, 2003; Tzafrir, 2005; Bryson, Forth y Kirby, 2005; Mohr y Yoghi, 2008; Beltrán et al., 2008).

Si el empleado percibe que la organización apoya su conducta, se genera una actitud positiva hacia ésta, por ejemplo, a través de la satisfacción laboral, desempeño en el trabajo y compromiso organizacional (Aselage y Eisenberger, 2003). En caso contrario, puede desarrollar una actitud negativa y de menor desempeño en el lugar de trabajo, por ejemplo, con bajo rendimiento, menor satisfacción (Rhoades, Eisenberger y Armeli, 2001). De esta manera, la organización desarrolla una percepción en sus individuos sobre la compatibilidad de cumplir con el desarrollo económico y social de la empresa y la recompensa que van a obtener (Rhoades y Eisenberger 2002; Shore \& Shore, 1995)

Se ha utilizado una adaptación de la escala propuesta por Eisenberger et al., (1986). Estos autores utilizan el término "apoyo organizativo percibido" para describir el grado en que los trabajadores consideran que el modelo de gestión les proporciona los medios y la información necesaria para realizar de forma adecuada su trabajo, valora su aportación y se preocupa por su bienestar. La escala original es de 36 indicadores pero existen estudios posteriores que reducen significativamente este número por tratarse del mismo factor (Eisenberger et al., 1990; Lee y Miller, 1999).

Los indicadores son los siguientes:

- se ponen en marcha acciones para identificar las necesidades de recursos e información de todo el personal

- la empresa comunica formalmente su política y estrategia a todos sus empleados 
- la dirección comunica y reconoce formalmente los logros de sus colaboradores

- se establecen procedimientos para conocer la opinión y satisfacción de nuestros empleados

- directivos y supervisores permiten a los empleados tener una alta autonomía y autocontrol sobre sus actuaciones

\section{HIPÓTESIS}

Ho: Las empresas que cuentan con un diseño organizativo flexible, unido a prácticas de alto rendimiento, generan un mayor nivel de compromiso entre los empleados y con la organización.

\section{METODOLOGÍA}

\subsection{Población y muestra}

Para comprobar la hipótesis y elaborar el presente estudio empírico, se han seleccionado aquellas empresas que tienen un reconocimiento positivo en el mercado sobre las prácticas de recursos humanos y de diseño organizativo que llevan a cabo. Se han escogido como muestra aquellas empresas que pertenecen al listado elabo-

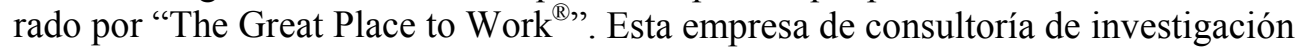
y gestión, publica a nivel mundial y de manera anual un listado de empresas que destacan por su gestión de personal, siendo calificadas como las mejores empresas para trabajar. A su vez, las empresas que aparecen en sus listados, son las que en comparación con otras, mejor beneficio económico han obtenido en los últimos 10 años (Great Place to Work, 2010).

Los factores que se evalúan para valorar la gestión de sus recursos humanos, giran en torno a la calidad del entorno de trabajo según la relación entre los empleados y directivos, la relación entre los empleados y su trabajo y con la empresa, y la relación entre los mismos empleados, obtienen una puntuación y son clasificadas por orden de mayor a menor.

La noción de llegar a ser un gran lugar para trabajar ha sido rápidamente reconocida por líderes en diversas industrias, organizaciones e instituciones educativas como un medio básico para mejorar los ambientes de los lugares de trabajo y, al hacerlo, su imagen y resultado económico. Las prácticas de alto rendimiento evaluadas se sustenta que son constantes en las empresas categorizadas por Great Place to Work.

Por tanto, partiendo del listado de las mejores empresas donde trabajar, se solicitó información a todas las 10 primeras empresas de cada país de América del Sur: Brasil (subdivido en función de las zonas y sector empresarial), Colombia, Perú, Argentina (subdividida en varios bloques en función del tamaño empresarial), Bo- 
livia, Chile, Méjico, Ecuador, Centroamérica y Paraguay, lo que supuso un total de 200 empresas, dirigiéndonos a los Directores de Recursos Humanos y Directores Generales.

La información se ha obtenido a través de una entrevista semiestructurada, la observación directa de dichas empresas y diversos documentos. Se ha recogido información por varias fuentes, personas y sitios, con el fin de reducir el riesgo a reflejar solamente las predisposiciones o carencias que puede tener un único método, lo que permite una mejor evaluación de la información, así como una validación y generalización de los resultados (Maxwell, 1996).

Del total de empresas contactadas, se recibió respuesta de 38, siendo finalmente evaluadas a través de entrevistas en profundidad y posteriormente analizadas 15 empresas realizando el estudio en las siguientes: Great Place to Work, Femsa Coca Cola, Yanbal y SC Johnson en Colombia; Profuturo, R.E.P., JV Resguardo y Novartis Pharma en Perú; Pormade, Semco y Bradesco en Brasil; Accor Services, Hotel Ibis y Young and Rubicam, en Uruguay y Exhacta en Argentina.

\subsection{Medición de Variables y Análisis}

Para desarrollar la entrevista se elaboró una encuesta formada por las prácticas de alto rendimiento, variables organizativas y compromiso de los empleados, siguiendo la escala propuesta por Likert. El encuestado debía señalar su grado de acuerdo o desacuerdo con cada variable, otorgando a cada respuesta una puntuación favorable o desfavorable. La justificación razonada de tales puntuaciones totales, admite diversos grados de aprobación, frecuencias que tipifican la ocurrencia o no de hecho y perfila el comportamiento de general de los evaluados. La probabilidad de acuerdo o desacuerdo con cualquiera de las series de ítems favorables o desfavorables, con respecto a un objeto, varía directamente con el grado de actitud de los individuos en las organizaciones. Se han recogido un total de 3 ítems, repartidos en 30 variables, prácticas de alto rendimiento de los empleados, variables de diseño organizativo y compromiso de los empleados:

- Prácticas de alto rendimiento: contratación selectiva, contratación fija, formación intensiva, formación genérica, promoción cualitativa, evaluación cualitativa, retribución basada en el individuo, retribución variable, retribución media-alta, retribución a largo plazo, uso de incentivos colectivos.

- Variables organizativas. Centralización: autonomía, ámbito de control del director, número de supervisores directos, ratio de supervisión/ trabajador, jerarquía de autoridad, jerarquía de control, número de niveles verticales, participación personal en la toma de decisiones y participación departamental. Con estas se pretende evaluar el grado de flexibilidad existente en las organizaciones y las relaciones descritas entre a estructura organizacional y el comporta- 
miento de los individuos. Formalización: estructuración de actividades, nivel de comunicación escrita, reglas y procedimientos. Y especialización: diversidad de funciones y número, número de categorías laborales, di visión del trabajo.

- El compromiso con los empleados: Acciones de identificación de necesidades de recursos e información, comunicación y reconocimiento de los logros de los empleados, comunicación de la política y la estrategia empresarial, conocimiento de la opinión y satisfacción de los empleados, empleados con gran autonomía y autocontrol.

\subsection{Resultados}

Siguiendo el estudio de Wang et. al (2003) y mediante un análisis cluster, se han clasificado las empresas de la muestra en función de las prácticas de alto rendimiento, diseño organizativo y compromiso con los empleados que llevan a cabo.

Para ello, se han empleado los programas informáticos SPSS, SPAD, encontrando dos grandes grupos de empresas. Un primer grupo que se encuentra en una fase inicial en el logro de un alto compromiso de sus empleados, y un segundo grupo que está en una fase más avanzada. La diferencia está en función de la forma en que aplican en sus empresas las variables de diseño organizativo y de alto rendimiento.

Gráfico 1: Grupos de empresas

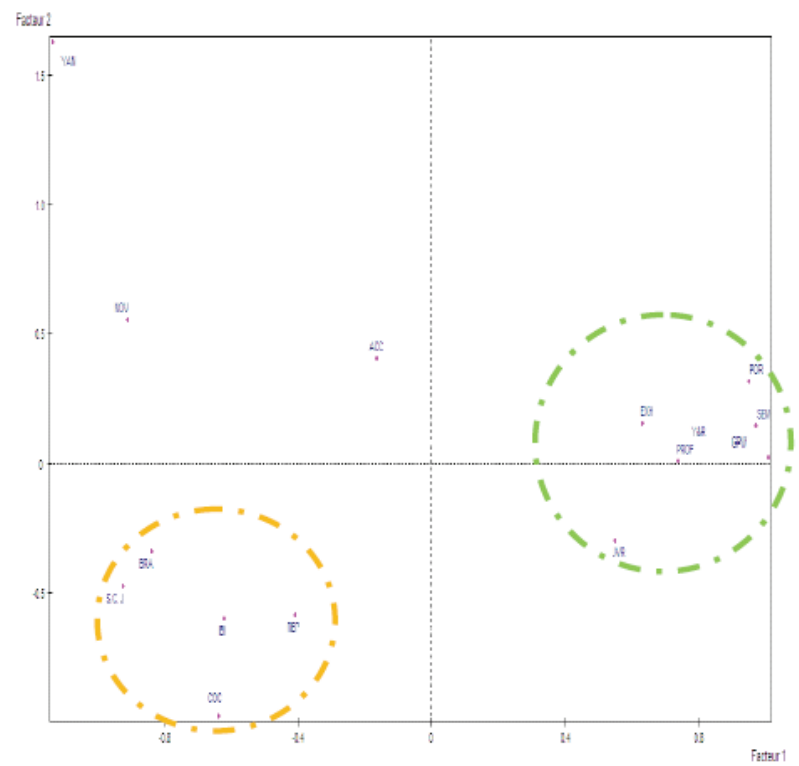

Fuente: Elaboración propia a partir de datos obtenidos en la investigación 
Como se puede observar de manera gráfica, además de los dos grupos que pueden apreciarse, aparecen dos excepciones, que se corresponden a las empresas Novartis y Accor. Por su parte, Novartis se caracteriza por ser una empresa con un diseño organizativo muy clásico, un elevado número de categorías laborales, alto nivel de jerarquía, y en definitiva, pocas prácticas de alto rendimiento. En cambio, Accor apuesta más por la innovación y desarrollo de nuevas prácticas de diseño organizativo, pero acompañado por un alto nivel de dependencia y centralización, sin autonomía y autogestión de los empleados y los equipos de trabajo.

\section{Grupo 1: En fase inicial del logro de un alto compromiso de los empleados}

Las empresas que componen el grupo 1 son: S.C. Johnson, IBIS, REP Perú, Bradesco, Coca Cola, Yanbal, Novartis y Bradesco.

Todas en general tienen la misma orientación al comportamiento de las variables calificadas y tienden en general a la caracterización de un solo perfil.

Es un grupo en fase o en proceso de conseguir de manera óptima un mayor compromiso por parte de sus empleados. Han logrado conseguir climas laborales óptimos y escenarios propicios para el desarrollo de prácticas de alto rendimiento en la dirección de talentos. Presentan un elevado concepto de autoridad y reconocimiento total de una sociedad del conocimiento basada en la confianza y en la capacidad individual de la autogestión, el autocontrol y el autodesarrollo. Pero también estas empresas se caracterizan por un muy alto control jerárquico, una retribución medio-baja, bajo uso de incentivos colectivos, baja autonomía, bajo número de empleados con autonomía y autocontrol, baja participación personal en la toma de decisiones, alta normalización y reglas, y un alto número de niveles verticales. En conclusión, podría decirse que son empresas que empiezan a incorporar nuevas propuestas organizacionales y dan mayor participación a nuevos cambios hacia el conocimiento y el crecimiento de los empleados y los procesos.

\section{Grupo 2: En fase avanzada del logro de un alto compromiso con los empleados}

Con este mismo objeto y orientación, se ha encontrado un segundo grupo compuesto por Profuturo, J.V. Resguardo, Exhacta, Great Place to Work, Young and Rubicam, Pormade y Semco. 
Tabla 3: Grupo 1, empresas en fase inicial

\begin{tabular}{|c|c|c|c|}
\hline VARIABLES SIGNIFICATIVAS & CLASIFICACIÓN & $\begin{array}{c}\text { GRADO DE } \\
\text { CUMPLIMIENTO }\end{array}$ & V. TEST \\
\hline Retribución medio-alto & Alto rendimiento & Medio & 2.54 \\
\hline Uso de incentivos colectivos & Alto rendimiento & Bajo & 2.54 \\
\hline Formación genérica & Alto rendimiento & Bajo & 1.16 \\
\hline Retribución a largo plazo & Alto rendimiento & Bajo & 1.16 \\
\hline Autonomía del empleado & Organizativa / centralización & Bajo & 1.63 \\
\hline Jerarquía de autoridad & Organizativa / centralización & Muy alto & 1.63 \\
\hline $\begin{array}{l}\text { Participación del personal en la } \\
\text { toma de decisiones }\end{array}$ & Organizativa / centralización & Bajo & 1.63 \\
\hline $\begin{array}{l}\text { Número de Reglas y procedi- } \\
\text { mientos }\end{array}$ & Organizativa / formalización & Muy alto & 1.63 \\
\hline Jerarquía de control & Organizativa / centralización & Muy alto & 2.54 \\
\hline $\begin{array}{l}\text { Participación departamental en } \\
\text { la toma de decisiones }\end{array}$ & Organizativa / centralización & Bajo & 1.63 \\
\hline Número de categorías laborales & Organizativa / especialización & Muy alto & 1.63 \\
\hline Número de niveles verticales & Organizativa / centralización & Alto & 1.38 \\
\hline $\begin{array}{l}\text { Diversidad de funciones y } \\
\text { número }\end{array}$ & Organizativa / especialización & Medio & 1.38 \\
\hline División del trabajo & Organizativa / especialización & Muy alto & 1.28 \\
\hline $\begin{array}{l}\text { Conocimiento de la opinión y } \\
\text { satisfacción de los empleados }\end{array}$ & Compromiso & Alto & 1.63 \\
\hline $\begin{array}{l}\text { Empleados con gran autonomía } \\
\text { y autocontrol }\end{array}$ & Compromiso & Bajo & 1.63 \\
\hline $\begin{array}{l}\text { Acciones de identificación de } \\
\text { necesidades y recursos }\end{array}$ & Compromiso & Medio & 1.63 \\
\hline
\end{tabular}

Fuente: Elaboración propia a partir de datos obtenidos en la investigación

Se caracterizan por llevar a cabo prácticas de alto rendimiento unidas a las variables organizativas que permiten alcanzar un mayor compromiso por parte de los empleados: elevada participación de los trabajadores en la toma de decisiones (de manera individual, no departamental), alto conocimiento de la opinión y satisfacción de los empleados, empleados con gran autonomía y autocontrol, un alto uso de incentivos colectivos, bajo número de categorías laborales, contratación selectiva, alta formación, características de retribución medio alto basada en los individuos, con tendencia al largo plazo, junto con baja jerarquía, formación genérica y muy bajo ámbito de control del director. Son empresas que se basan autogestión y el autocontrol de sus individuos, alejándose de la supervisión y permitiendo climas organizacionales más proactivos e informales, atendiendo al conocimiento y a la pertinencia de los resultados más que a los requisitos y a la dependencia de los procesos. 
Tabla 4: Grupo 2, empresas en fase avanzada

\begin{tabular}{|c|c|c|c|}
\hline VARIABLES SIGNIFICATIVAS & CLASIFICACIÓN & $\begin{array}{c}\text { GRADO DE } \\
\text { CUMPLIMIENTO }\end{array}$ & V. TEST \\
\hline Uso de incentivos colectivos & Alto rendimiento & Alto & 2.99 \\
\hline Contratación Selectiva & Alto rendimiento & Muy alto & 2.99 \\
\hline Formación intensiva & Alto rendimiento & Muy alto & 2.54 \\
\hline Retribución medio-alto & Alto rendimiento & Muy alto & 2.46 \\
\hline $\begin{array}{l}\text { Retribución basada en el } \\
\text { individuo }\end{array}$ & Alto rendimiento & Muy alto & 2.37 \\
\hline Retribución a largo plazo & Alto rendimiento & Alto & 1.95 \\
\hline Formación genérica & Alto rendimiento & Muy alto & 1.86 \\
\hline Contratación fija & Alto rendimiento & Muy Bajo & 1.63 \\
\hline $\begin{array}{l}\text { Participación personal en la toma } \\
\text { de decisiones }\end{array}$ & Organizativa / centralización & Muy alto & 3.61 \\
\hline Jerarquía de control & Organizativa / centralización & Bajo & 1.95 \\
\hline $\begin{array}{l}\text { Número de categorías labora- } \\
\text { les }\end{array}$ & Organizativa / especialización & Muy bajo & 2.99 \\
\hline Ámbito de control del director & Organizativa / centralización & Muy Bajo & 1.43 \\
\hline $\begin{array}{l}\text { Conocimiento de la opinión y } \\
\text { satisfacción de los empleados }\end{array}$ & Compromiso & Muy alto & 3.61 \\
\hline $\begin{array}{l}\text { Empleados con gran autonomía } \\
\text { y autocontrol }\end{array}$ & Compromiso & Muy alto & 3.61 \\
\hline
\end{tabular}

Fuente: Elaboración propia a partir de datos obtenidos en la investigación

A las empresas del grupo 1 les une con gran relevancia variables de carácter organizativo, destacando el no haber logrado aún unos niveles óptimos en su utilización. Son empresas donde persiste una jerarquía alta y una autonomía baja. Prevalece el individuo frente al grupo, a través de la participación en la toma de decisiones y la importancia de los niveles jerárquicos. En este sentido, presentan demasiados niveles jerárquicos y excesivas categorías profesionales. Incidiendo en el grado de cumplimiento de estas variables de diseño organizativo hacia una mayor flexibilización de las mismas, llegarían a una fase de compromiso más avanzada con los empleados, como ocurre en el grupo de empresas 2. Por ello, son empresas con un diseño organizativo que presentan un grado bajo de flexibilidad.

A diferencia del anterior, el grupo 2 se sitúa en una fase avanzada en el logro del compromiso con los empleados. La jerarquía de control y el número de categorías laborales presentan un grado de cumplimiento bajo o muy bajo. La libertad de actuación de los empleados, la autogestión, es una práctica bastante habitual, unido a una alta participación de los empleados en la toma de decisiones. Por tanto, el grupo se caracteriza por haber conseguido unos grados de cumplimiento elevados con respecto al diseño organizativo flexible y las principales prácticas de alto rendimiento, de tal manera que, ante empresas que son flexibles en su diseño, logran un mayor compromiso.

Por tanto, las variables de diseño organizativo, afectan de manera muy significativa al grado de avance en el logro del compromiso con los empleados, apoyando 
a las prácticas de alto rendimiento. De esta manera, las empresas que se encuentran en una fase inicial, necesitan realizar un esfuerzo mayor en los parámetros de: jerarquía de control, autonomía del empleado, reglas y procedimientos, participación departamental en la toma de decisiones, número de categorías laborales y diversidad de funciones, división del trabajo, y de manera muy destacada en la participación del personal en la toma de decisiones.

De esta manera, las variables organizativas junto con las prácticas de alto rendimiento apoyarían el logro de un mayor compromiso de la empresa con sus empleados justificando la hipótesis planteada en la presente investigación.

\section{CONCLUSIONES}

Las prácticas de alto rendimiento evaluadas son frecuentes en las empresas recogidas por Great Place to Work y todas las empresas estudiadas consiguen resultados aceptables en términos de compromiso de los empleados.

Se observa en todas ellas, cómo para lograr un mayor compromiso de los empleados, es necesario un diseño organizativo que sea flexible, donde las variables organizativas permitan a las empresas aplicar las prácticas de alto rendimiento.

Se ha constatado que las empresas que consiguen unos mejores resultados de compromiso, presentan estructuras organizativas flexibles, es decir, son organizaciones más planas, con un menor número de niveles jerárquicos, existencia de una importante disminución de la autoridad formal y un importante desarrollo de los equipos de trabajo. En definitiva, presentan una alta flexibilidad organizativa y de recursos humanos.

El grado de formalización de estas organizaciones, es cada vez más simple, destaca la disminución de las áreas delimitadas, existe un grado elevado de informalidad y se reconocen nuevas formas de trabajo, incrementando las competencias y las habilidades cognitivas.

También existe una amplia disposición de las organizaciones estudiadas hacia el cambio en torno a la especialización, entendiendo que la realización de una o de unas pocas funciones pueden limitar la capacidad de los individuos en las organizaciones. Aparecen nuevos conceptos en el diseño de los puestos de trabajo, en especial empiezan a desdibujarse sus límites funcionales y aparece el trabajo en equipo, pero además de manera más flexible y autogestionada.

Ante esta situación se requieren individuos más comprometidos. Los empleados gozan de plena libertad para sugerir cambios y proponer nuevos procesos, para cambiar las organizaciones según las necesidades que imponen las condiciones del entorno, tanto como sea necesario para hacerla adaptativa y flexible a las nuevas condiciones y enfrentarse a la internacionalización y globalización de la economía.

Por ello se hace necesario que en las organizaciones se comparta toda la información, se reconozcan los logros alcanzados, se conozca y comparta la política y la 
estrategia empresarial, se conozca la satisfacción de los empleados y éstos tengan una gran autonomía y autogestión.

Las organizaciones cada vez se comprometen más con su recurso humano, se relacionan de forma más directa con las necesidades de su cliente interno y se convencen cada vez más de que sus resultados son, en una parte importante, consecuencia del clima organizacional conseguido a través de prácticas del alto rendimiento que son de carácter flexible, la flexibilidad el diseño organizativo, la interacción entre el compromiso de los empleados y la organización y la adecuación de una infraestructura dispuesta y disponible para crecer.

Las prácticas de recursos humanos adoptadas por la organización pueden considerarse como una capacidad complementaria al diseño organizativo flexible y por tanto contribuir al desarrollo de ventajas competitivas sostenibles.

El estudio empírico de este trabajo demuestra, por tanto, que el diseño organizativo flexible interviene de una manera determinante en la relación entre las prácticas de alto rendimiento y el compromiso de los empleados.

\section{BIBLIOGRAFÍA}

AHMED, P.K.; RAFIQ, M. y SAAD, N.M. (2003). "Internal marketing and the mediating role of organizational competencies". European Journal of Marketing, 37, $\mathrm{n}^{\circ}$ 9, pp.1221-1241.

APPELBAUM, E. and BATT, R. (1994) The New American Workplace: Transforming Work Systems in the United States. Ithaca, NY: ILR Press.

APPELBAUM, E., BERG, P. and KALLEBERG, A. L. (2000). Manufacturing Advantage: Why High-Performance Work Systems Pay Off. Ithaca, NY. Cornell University Press.

ARGYRIS, C y SCHÖN, D.A. (1996). Organizational learning II: Theory, method and practice, Reading, PA. Addison-Wesley.

ARTHUR, J.B. (1994). "Effects of human resource Systems on manufacturing performance and turnover", Academy of Management Journal, n 37, pp. 670687.

ASELAGE, J. y EISENBERG, R. (2003). Perceived organizational support and psychological contracts: A theoretical integration, Journal of Organizational Behavior, 24: 491-509

ATKINSON, J. (1984). The Flexible Firm and the Shape of Jobs to Come. (Labour Market Issues, 5). Oxford.

BAE, J. y LAWLER, J.J. (2000). "Organizational and HRM strategy in Korea: impact on firms performance in an emerging economy", Academy of Management Journal, 43, $\mathrm{n}^{\circ} 3$, pp. 502-517.

BAHRAMI, H. (1992). 'The Emerging Flexible Organization: Perspectives from Silicon Valley', California Management Review, 34, p. 33. 
BAIRD, L.L. y MESHOULAM, I. (1988). "Managing two fits of strategic human resource management", Academy of Management Review, 13, $\mathrm{n}^{\circ}$ 1, pp. 116-128.

BATT, R. (2000). "Strategic segmentation in front-line services: matching customers, employees and human resource systems", International Journal of Human Resource Management, 11, $\mathrm{n}^{\circ}$ 3, pp. 540-561.

BAYO MORIONES, A. y MERINO DÍAZ DE CERIO, J. (2002). "Las prácticas de recursos humanos de alto compromiso: un estudio de los factores que influyen sobre su adopción en la industria española". Cuadernos de Economía y Dirección de la Empresa, $\mathrm{n}^{\mathrm{o}}$ 12, pp. 227-246.

BAYO, A. y MERINO, J. (2001). "Quality management and high performance work practices: do they coexist? International Journal of Production Economics, 73, nº 3, pp. 251-260.

BAYONA, C., S. GOÑI y C. MADORRÁN (1999). "Compromiso organizacional: implicaciones para la gestión estratégica de los recursos humanos". Revista Europea de Dirección y Economía de la Empresa, pp. 139-149.

BECKER, B. y GERHART (1996). "The impact of human resource management on organizational performance: progress and prospects", Academy of Management Journal, vol. 39, pp. 779-801.

BELTRÁN MARTÍN, I;, ROCA PUIG, V.; ESCRIG TENA, A. y BOU LLUSAR, J.C. (2008). "Human resource flexibility as a mediating variable between high performance work systems and performance". Journal of Management, 34, no 5, pp. 1009-1044.

BHATTACHARYA, M.; GIBSON, D.; DOTTY, D. (2005). "The Effects of Flexibility in Employee Skills, Employee Behaviors, and Human Resource Practices on Firm Performance", Journal of Management, 31(4), pp. 622-640.

BISHOP, J.W.; SCOTT, K.D. y BURROUGS, S.M. (2000). "Support, commitment and employee outcomes in a team environment", Journal of Management, 26, pp. 1113-1132.

BLACKBURN, R. y ROSEN, B. (1993). "Total quality and human resources management: lessons learned from Baldrige Award-winning companies", Academy of Management Executive, 7 (3), pp. 49-66.

BLAU, G.J. y BOAL, K.R. (1987). "Conceptualizing how job involvement and organizational commitment affect turnover and absenteeism", Academy of Management Review, $12, \mathrm{n}^{\circ}$ 2, pp.288-300.

BOXALL, P. y STEENEVELD, M. (1999). "Human resource strategy and competitive advantage: a longitudinal study of engineering consultancies", Journal of Management Studies, vol. 36, $\mathrm{n}^{\circ}$ 4, pp. 443-463.

BRYSON, A.; FORTH, J. y KIRBY, S. (2005). "High-involvement management practices. Trade Union Representation and workplace performance in Britain". Scottish Journal of Political Economy, $\mathrm{n}^{\circ}$ 52, pp. 451-491.

CAMISÓN, C. y VILLAR, A. (2010). "Análisis del papel mediador de las capacidades de innovación tecnológica en la relación entre la forma organizativa 
flexible y el desempeño organizativo". Cuadernos de Economía y Dirección de la Empresa, 45, pp. 115-144.

CAPPELLI, P y CROCKER-HEFTER, A. (1996). "Distinctive human resources are firm's core competencies", Organizational Dynamics, 24 (3), pp. 7-22.

CAPELLI, P. y NEUMARK, D. (2001). "Do high-performance work practices improve establishment level outcomes?" Industrial and Labour Relations Review, 54, no 4, pp. 737-775.

CAPPELLI, P y NEUMARK, D. (2001). "External job churning and internal job flexibilitly" Unpusblished paper, Wharton School of the University of Pennsylvania.

CARDY, R.L. y ROBBINS, G.H. (1996). "Human Resource Management in a Total Quality Organizational Environment: Shifting from a Traditional to a TQ HRM Approach", Journal of Quality Management, (11), pp. 5-20.

CASTELLS, M. (1996) The rise of the network society; The information age: Economy, society and culture. Blackwell, Malden MA.

CÉSPEDES, J.J.; JEREZ, P. y VALLE, R. (2005). "Las prácticas de Recursos Humanos de Alto Rendimiento y la capacidad de aprendizaje organizativo: incidencia e implicaciones". Cuadernos de Economía y Dirección de la Empre$s a, \mathrm{n}^{\circ} 24$, pp. 29-56.

COLCLOUGH, G y TOLBERT, C. M (1992). Work in the Fast Lane: Flexibility Divisions of Labor and Inequality in High-Tech Industries. Albany. SUNY

COTTON, J. L. (1993). Employee Involvement: Methods for Improving Performance and Work Attitudes. Newbury Park, Cal.. Sage.

DANVILA, I. y SASTRE, M.A. (2009). "Human capital and sustainable comparative advantage: An analysis of the relationship between training and company results", International Entrepreneurship and Management Journal, vol. 5 (2), 139-163.

DANVILA, I.; SASTRE, M.A. Y RODRÍGUEZ, A. (2009). "The effect of training on the firm results in companies of services: a study with panel data", International Journal of Manpower, vol. 30 (4), 393-407.

DAVIS, K. y NWESTROM, J. (2000). Comportamiento humano en el trabajo. México: McGraw Hill.

DELANEY, J. T. and HUSELID, M. A. (1996). "The impac of human resource management practices on perceptions of organizational performance". Academy of Management Journal, 39: 949-69.

DELERY, J. y DOTY, H. (1996). "Modes of theorizing in strategic in strategic human resource management: test or universalistic, contingency and configurational performance predictions", Academy of Management Journal, vol. 39, $\mathrm{n}^{\circ}$ 4, pp. 802-835.

DELERY, J.E. (1998). "Issues of fit in strategic human resource management: implications for research", Human Resource Management Review, vol. 8, pp. 289-309. 
DEYO, F.C. (1997). "Labor and post-Fordist industrial restructuring in East and Southeast Asia", Work and Occupations, 24. 97-118

EISENBERGER, R., FASOLO, P. y DAVIS-LAMASTRO, V. (1990).’Perceived organizational support and employee diligence, commitment, and innovation" Journal of Applied Psychology, vol. 75, pp. 51-59

EISENBERGER, R.; HUNTINGTON, R.; HUTCHISON, S. y SOWA, D. (1986) "Perceived organizational support", Journal of Applied Psychology, vol. 71, pp. 500-507.

ENGLEHARDT, C. S. y SIMMONS, P. R. (2002). 'Organizational Flexibility for a Changing World', Leadership and Organization Development Journal, 23, pp. 113-122.

EVANS, P.A.L. y DOZ, Y. (1993) Dualities, a paradigm for human resource and organizational development in complex multinationals. In: PUCIK, V., TICHY, N.M. and BARNETT, C.K. (eds.), Globalizing management. Wiley, New York.

EVANS, S. J. (1991). 'Strategic Flexibility for High Technology Manoeuvres. A Conceptual Framework', Journal of Management Studies, 28, pp. 69-89.

FERRIS, G.R.; HOCHWARTER, W.A.; BUCKLEY, M.R.; HARRELL-COOK, G. y FRINK, D.D. (1999). "Human resources management: some new directions", Journal or Management, vol. 25, pp. 385-415.

FORD, J.D. (1979). Institutional versus questionnaire measures of organizational structure: A reexamination. Academy of Management Joumal, vol. 22, pp.601610.

GONZÁlEZ ÁlVAREZ, N. y NIETO ANTOLÍN, M. (2007). "El papel de la ambigüedad causal como variable mediadora entre las prácticas de recursos humanos de alto compromiso y los resultados corporativos". Revista Europea de Dirección y Economía de la Empresa, 16, no 4, pp. 107-126.

GUEST, D.E. (1987). "Human resource management and industrial relations". Journal of Management Studies, 24, no 5, pp. 503-521.

GUITTLEMAN, M., HORRIGAN, M. and JOYCE, M. (1998). '"Flexible" workplace practices: evidence from a nationally representatiave survey'. Industrial and Labor Relations Review, 52: 99-113.

GUTHRIE, J.P. (2001). "High-involvement work practices, turnover, and productivity: evidence from New Zealand". Academy of Management Journal, 44, $\mathrm{n}^{\mathrm{o}} 1$, pp.180-190.

GUTHRIE, J.P.; SPELL, C.S. y NYAMORI, R.O. (2002). "Correlates and consequences of high involvement work practices: the role of competitive strategy". International Journal of Human resource Management, 13, $\mathrm{n}^{\circ}$ 1, pp. 183197.

HAGE, J. y R. DEWAR (1973). 'Elite Values versus Organizational Structure in Predicting Innovation', Administrative Science Quarterly, 18, pp. 279-290

HAGE, J.; y AIKEN, M (1967). Relationships of centralization to other structural properties. Administrative Science Quarterly, vol. 69, 32-40. 
HASTINGS, C. (1993) The new organization. McGraw Hill, New York.

HUNTER, L., MCGREGOR, A., MACINNES, J. y SPROULL, A. (1993). "The "flexible firm": strategy and segmentation", British Journal of Industrial Relations, 31: 387-407

HUSELID, M.A. (1995). "The impact of human resource management practices on turnover, productivity, and corporate financial performance". Academy of Management Journal, $\mathrm{n}^{\mathrm{o}}$ 38, pp. 673-703.

HUSELID, M.A. y BECKER, B.E. (1996). "Methodological Issues in crosssectional and panel estimates of the human resource-firm performance link", Industrial Relations, 35, n⿳0 3, pp. 400-422.

HUSELID, M.A., JACKSON, S.E. y SCHULER, R.S. (1997). “Technical and strategic human resource management effectiveness as determinants of firm performance", Academy of Management Journal, 40, nº 1, pp. 171-188.

ICHNIOWSKI, C., KOCHAN, T.A.; LEVINE, D., OLSON, C., y STRAUSS, G. (1996). "What works at work: overview and assessment", Industrial Relations, vol 35, pp. 352-332

ICHNIOWSKI, C.; SHAW, K y PRENNUSHI, G. (1997). " The effects of human resource management practices on productivity: A study of steel finishing lines", American Economic Review, 87, n 3, pp. 291-313.

ILES, P.; FORSTER, A. y TINLINE, G. (1996). "The changing relationships between work commitment, personal flexibility and employability: an evaluation of field experiment in executive development", Journal of Managerial Psychology, 42, pp. 727-786.

JARILLO, J.C. (1993) Strategic networks; creating the borderless organization. Butterworth Heinemann, Oxford.

KAMOCHE, K. (1996). "Strategic human resource management within a resourcecapability of the firm", Journal of Management Studies, 33, pp. 213-233.

KARUPPAN, C. (2004). "Strategies to Foster Labor Flexibility", International Journal of Productivity and Performance Management, 53 (6), pp. 532-547.

KERR, J.L., and JACKOFSKY, E.F. (1989), 'Aligning Managers With Strategies: Management Development versus Selection,' Strategic Management Journal, $10,157-170$

KIM, L. (1998). "Crisis construction and organizational learning: capability building in catching-up at Hunday Motor", Organization Science, 9 (4), pp. 506-521.

KOFMAN, F. y SENGE, P.M. (1993). "Communities of commitment: the heart of learning organizations", Organizational Dynamics, vol.22, no2, pp.4-23

LAWLER, E. E. III (1988). "Choosing an involvement strategy". Academy of Management Executive, 2: 197-204.

LAWLER, E.E. (1992). The ultimate advantage: creating the high-involvement organization. San Francisco. Jossey- Bass. 
LEE, J. y MILLER, D. (1999). "People matter: commitment to employees, strategy and performance in korean firms", Strategic Management Journal, vol. 20, pp. 579-593.

LENGNICK-HALL, C.A. y LENGNICK-HALL, M.L. (1988). "Strategic human resource management: a review of the literature and a proposed typology", Academy of management Review, vol. 13, 3, pp. 454-470.

LEONARD-BARTON, D.(1992). "The factory as a learning laboratory", Sloan Management Review, pp. 23-38.

MACDUfFIE, J. (1995). "Human Resource Bundles and Manufacturing Performance: Organizational Logic and Flexible Production Systems in the World Auto Industry", Industrial and Labor Relations Review, 48 (2), pp. 197-221.

MACDUFFIE, J. P. (1995). "Human resources bundles and manufacturing performance: organizational logic and flexible production systems in the world auto industry". Industrial and Labor Relations Review, 48. 197-221.

MARCHINGTON, M. y GRUGULIS, I. (2000). "Best practice human resource management: perfect opportunity or dangerous illusion?", The International Journal of Human Resource Management, vol 11, nº, pp. 1104-1121

MARTÍ AUDÍ, N. (2008). "High commitment human resource practices in spanish call centres. Nature, sources and effects on voluntay turnover". Tesis Doctoral Universitat Rovira i Virgili

MARTÍNEZ, Á., VELA, M.J, PÉREZ, M. y DE LUIS, P. (2011). “Innovación y flexibilidad de recursos humanos: el efecto moderador del dinamismo del entorno"Revista Europea de Dirección y Economía de la Empresa, vol. 20, núm. 1 (2011), pp. 41-68

MCGREGOR, D. (1960). The human side of enterprise, McGraw-Hill, New York

MCNABB, R. and WHITFIELD, K. (1998) 'The Impact of Financial Participation and Employee Involvement on Financial Performanee', Scottish Journal of Political Econotny, 45(2). 171-87.

MEYER, J. P. y N. J. ALLEN (1997). Commitment in the Workplace. Theory, research and application. Thousand Oaks, California. Sage publications, inc.

MILLIAM J.; VON GLINOW, M.; NATHAM, M. (1991). "Organizational Life Cycles and Strategic International Human Resource Management in Multinational Companies: Implications for Congruence Theory", Academy of Management Review, 16 (2), pp. 318-339.

MOHR, R.D. y ZoGHI, C. (2008). "The high-involvement work design and job satisfaction". Industrial and Labor Relations Review, 61, n 3, pp. 275-296.

NICHOLSON, N., REES A. and BROOKS-ROONEY A. (1990). 'Strategy, Innovation and Performance', Journal of Management Studies, vol. 27, pp. 511-534.

NOHRIA, N. and GHOSHAL, S. (1997) The differentiated network. Organizing multinational corporations for value creation. Josey Bass, San Fransisco.

NONAKA, I, y TAKEUCHI, H. (1995). The knowledge-creating company, NY. Oxford University Press. 
O'DELL, C. (1996). "A current review of knowledge management best practice", Knowledge Management 96 Conference, Business intelligence, Londres.

OAKLAND, J.S.- (2004). Oakland and Quality Management, Elsevier Butterworth-Heinemann, Burlington, MA

OECD (1999). Employment Outlook. Paris. Organisation for Economic Cooperation and Development.

ORDIZ Fuertes, M. y Fernández Sánchez, E. (2003). "High-involvement practices in human resource management: concept and factors that motive their adoption". International Journal of Human Resource Management, 14, $\mathrm{n}^{\mathrm{o}}$ 4, pp. 511529.

ORDIZ, M. (2002). "Prácticas de alto rendimiento en recursos humanos: concepto y factores que motivan su adopción", Cuadernos de Economía y Dirección de la Empresa, vol. 12, pp. 247-265

ORDÓNEZ, P. (2005). "Gestión estratégica de recursos humanos, flexibilidad organizativa y encaje estratégico: Implicaciones para la competitividad empresarial", ICADE, Revista Cuatrimestral de las Facultades de Derecho y Ciencias Económicas y Empresariales, $\mathrm{n}^{\circ} 65,31-74$

OSTERMAN, P. (1994). "How common os workplace transformation and who adopts it?". Industrial and Labor Relations Review, n ${ }^{\circ} 47$, pp. 173-188.

OSTERMAN, P. (2000). "Work reorganization in an era of restructuring: trends in diffusion and effects on employee welfare". Industrial and Labor Relations Review, 53. 179-96.

PENNINGS, J.(1973). Measures of organization structure: A methodological note. American Journal of Sociology, vol. 79,pp. 686-704,

PERROW, C. (1970). Organizational analysis: A sociological view. Wadsworth, Belmont, CA.

PFEFFER, J. (1994). "Competitive advantage through people", California Management Review, 36, $\mathrm{n}^{\mathrm{o}} 2$, pp. 9-28.

PFEFFER, J. (1998). La ecuación humana, Ediciones Gestión 2000, Barcelona

POWELL, I; MONTGOMERY, M. y COSGROVE, J. (1994). "Compensation structure and establishment quit and fire rates", Industrial Relations, vol. 33, pp. 229-248.

PUGH, D.S.; HICKSON, D.J.; HININGS, C.R.; y TURNER, C (1968). Dimensions of organization structure. Administrative Science Quarterly, vol. 13, 65105.

RHOADES, L., EISENBERG, R. y ARMELI, S. (2001). Affective commitment to the organization: The contribution of perceived organizational support. Journal of Applied Psychology, vol 86. 825-836

RHOADES, L., y EISENBERGER, R. (2002). Perceived organizational support: A review of the literature, Journal of Applied Psychology, vol. 87. 698-714. 
RICHARD, O.C. y JOHNSON, N.B. (2001). "Strategic Human Resource Management Effectiveness and firm performance". The International Journal of Human resource Management, $\mathrm{n}^{\circ}$ 12, pp. 299-310.

ROCA PUIG, V., ESCRIG TENA, A.B. y BOU LLUSAR, J.C. (2002). “Compromiso con los empleados y estrategia competitiva: un análisis intersectorial de su repercusión en los resultados". Cuadernos de Economía y Dirección de la Empresa, № 12 , pp. 267-289.

ROCHE, W. K. (1999). "In search of commitment-oriented human resource management practices and the conditions that sustain them", Journal of Management Studies, vol. 36, pp.653-678

SÁNCHEZ QUIRÓS, I (2002). "Un análisis de las medidas de estructuras organizativas: estructura diseñada frente a estructura emergente, Cuadernos de Estudios Empresariales, vol. 12, pp.271-291

SÁNCHEZ QUIRÓS, I. (2004). "The creation of employee commitment in Spanish hotels through cultural practices". Management Research, 2, no 2, pp. 175192.

SANZ VALLE, R. y SABATER SÁNCHEZ, R. (2002). “Enfoque universalista de recursos humanos. Estudio empírico de sus efectos sobre los resultados de la empresa", Revista Europea de Dirección y Economía de la Empresa, 11, nº 1, pp. 163-182.

SASHKIN, M. (1984). "Participative management is an ethical imperative", Organizational Dynamics, vol.12, nº 4 , pp.5-22

SATHE, V .(1978). Institutional versus questionnaire measures of organizational structure. Academy of Management Joumal, , vol 21, pp. 227-238.

SCHNEIDER, B. y BOWEN, D.E. (1993). "The service organizations human resource management is crucial", Organizational Dynamics, vol. 21, no 4, pp. 3952.

SCHOEMAKER, M. (1998) Organizeren van werk en contractrelaties; tussen slavernij en anarchie. Kluwer, Deventer.

SCHOEMAKER, M. (2003). "Identity in Flexible Organizations: Experiences in Dutch Organizations", Creativity and Innovation Management, vol.12, $\mathrm{n}^{\circ} 4, \mathrm{pp}$. 191-201

SCHULER, R.S., y JACKSON, S.E. (1987a). "Linking competitive strategy with human resource management practices", Academy of Management Executive, vol.1, (3), pp. 207-219.

SCHULER, R.S., y JACKSON, S.E. (1987b). "Organizational strategy and organization level as determinants of human resource management practices", Human Resource Planning, vol. 10 (3), pp. 125-141.

SHORE, L. M., y SHORE, T. H. (1995). "Perceived organizational support and organizational justice" In R. S. Cropanzano y K. M. Kacmar (Eds.), Organizational politics, justice, and support: Managing the social climate of the workplace, 149-164. Westport, CT. Quorum. 
SMITH, V. (1994). "New forms of work organization", Annual Review of Sociology, 23. 315-329

SNELL, S.A., YOUNDT, M.A., and WRIGHT, P.M. (1996), 'Establishing a Framework for Research in Strategic Human Resource Management: Merging Resource Theory and Organizacional Learning,' in Research in Personnel and Human Resources Management, ed. G.R. Ferris, Greenwich, CT. JAI Press, pp. $61-90$.

SNOW, C.C y SNELL, S.A (1993). "Staffing as strategy", en N. Schimitt y W. Borman (eds). Personnel selection in organizations, San Francisco. Jossey-Bass, pp. 448-479

TAYLOR, S., LEVY, O., BOYACIGILLER, N.A. Y BEECHLER, S. (2008). "Employee commitment in MNCs: Impacts of organizational culture, HRM and top management orientations". The International Journal of Human Resource Management, $19, \mathrm{n}^{\circ} 4$, pp. 501-527.

THE GREAT PLACE TO WORK (2010). http://www.greatplacetowork.es/best/index.php

TRUSS, C., GRATTON, L., HOPE-HAILEY, V., MCGOVERN, P. y STILES, P. (1997). "Soft and hard models of human management: a reappraisal", Journal of Management Studies, vol. 34, pp.53-73

TSAI, Y. Y WU, S. (2006). "Internal Marketing, Organizational Commitment and Service Quality". Service Systems and Service Management, 2, n ${ }^{\circ}$ 25/27, pp. 1292-1298.

TSUI, A.S, PEARCE, J.L., PORTER, L.W y HITE J.P. (1995). "Choice of employee-organization relationship: influence of external and internal organizational factors", Research in Personnel and Human Resources Management, 13. $117-51$

TZAFRIR, S.S. (2005). "The relationship between trust, HRM practices and firm performance". Internacional Journal of Human Resource Management, 16, no 9, pp. 1600-1622.

ULRICH, D. (1998). "Intellectual capital = competente x commitment", Sloan Management Journal, vol. 4, pp. 15-26

UPTON, D. (1995). "What Really Makes Factories Flexible?", Harvard Business Review, 73 (4), pp. 74-84.

VALLE, R.; MARTÍN, F.; ROMERO, P.M. y DOLAND, S.L. (2000). "Business strategy work processes and human resource training: are they congruence?", Journal of Organizational Behaviour, vol. 21, pp.283-297.

VOLBERDA, H. (1996). "Toward the Flexible Form: How to Remain Vital in Hypercompetitive Environments",

VOLBERDA, H. W. (1997), "Building flexible organizations for fast-moving markets", Long Range Planning, vol. 30, núm. 2, pp. 169-183.

VOLBERDA, H. W. (1999). Building the Flexible Firm. Oxford University Press, Oxford. 
WALTON, E.J. (1981). "The comparison of measures or organization structure ", Academy of Management Journal, vol. 6, pp. 155-160.

WALTON, R. E. (1985). "From control to commitment in the workplace". Harvard Business Review, 65. 77-84.

WANG, D; TSUI, A; ZHANG, y MA, L (2003). "Employment relationship and firm performance: evidence from an emerging economy", Journal of Organizational Behaviour, 24; pp. 511-535

WILLIAMS, A.P. (2001). "A belief-focused process model of organizational learning", Journal of Management Studies, 38 (1), pp. 67-85.

WOOD, S. (1999). "Human resource management and performance". International Journal of Management Reviews, 1.367-413.

WOOD, S. y ALBANASE, M.T. (1995). "Can we speak of a high commitment management on the shop floor?", Journal of Management Studies, vol. 32, pp. $215-247$

WOOD, S. y de MENEZES, L.M. (2008). "Comparing perspectives on high involvement Management and organizational performance across the British economy". The International Journal of Human Resource Management, 19, $\mathrm{n}^{\circ} 4$, pp. 639-682.

WORD, S (1999). "Human resource management and performance", International Journal of Management Reviews, vol. 1, nº 4, pp. 367-413

WRIGHT, P. y SNELL, S. (1998). "Toward a Unifying Framework for Exploring Fit and Flexibility in Strategic Human Resource Management", Academy of Management Review, 23 (4), pp. 756-772.

WRIGHT, P.M.; SMART, D.L. y MCMAHAN, G.C. (1995). "Matches between human resource and strategy among NCAA basketball teams", Academy of Management Journal, 38, pp. 1052-1074.

YOUNDT, M.A.; SNELL, S.A.; DEAN, J.W., y LEPAK, D.P. (1996). "Human resource management, manufacturing strategy, and firm performance", Academy of Management Journal, vol. 39, pp. 836-866.

ZATZICK, C.D. e IVERSON, R.D. (2006). "High-involvement management and workforce reduction: competitive advantage or disadvantage?", Academy of Management Journal, 49, $\mathrm{n}^{\mathrm{o}}$ 5, pp. 999-1015. 